\title{
OSCILACIONES MACROECONÓMICAS DE HONDURAS Y COSTA RICA EN RELACIÓN A LAS ECONOMÍAS DE LOS PAÍSES DESARROLLADOS
}

\author{
Mario Roberto Acevedo Amaya, Universidad Nacional Autónoma de Honduras (UNAH), \\ Facultad de Ciencias Económicas, Administrativas y Contables (FCEAC), \\ Instituto de Investigaciones Económicas y Sociales (IIES), \\ Ciudad universitaria, edificio C2, primer piso, Tel/fax: (504) 2239-1849 \\ E-mail: macevedo@iies-unah.org
}

\section{RESUMEN}

La estabilidad económica de los países desarrollados no ha sido sostenible en el tiempo debido a los cambios económicos y políticos suscitados en el mundo, generando un efecto multiplicador para aquellas naciones cuya economía es dependiente a dichos países. En este sentido, se plantea un análisis de las oscilaciones económicas de Alemania, Estados Unidos y Hong Kong, como economías referente, en torno a las economías de Honduras y Costa Rica, a fin de conocer los puntos de giro, la similitud, las tendencias de las oscilaciones, periodos, rangos, desfases y desviaciones, considerando para ello como base; las variaciones del Producto Interno Bruto (PIB), PIB per cápita, superávit / déficit de efectivo, Deuda Externa y la Inversión Extranjera Directa, para un periodo de tiempo de diez y nueve años.

Palabras Clave: Ciclos Económicos, Globalización, Asimetrías Económicas, Dependencia Económica. 
Vol. 4

$\mathrm{N}^{\circ} 2$

2013

E\&A

IIES

104

\title{
MACROECONOMIC FLUCTUATIONS OF COSTA RICA AND HONDURAS ABOUT THE ECONOMIES OF DEVELOPED COUNTRIES
}

\author{
Mario Roberto Acevedo Amaya, Universidad Nacional Autónoma de Honduras (UNAH), \\ Facultad de Ciencias Económicas, Administrativas y Contables (FCEAC), \\ Instituto de Investigaciones Económicas y Sociales (IIES), \\ Ciudad universitaria, edificio C2, primer piso, Tel/fax: (504) 2239-1849 \\ E-mail: macevedo@iies-unah.org
}

\begin{abstract}
The economic stability of developed countries has not been sustainable along the time due to political events and economics changes raised in the world, producing a multiplier effect for nations whose economy dependent of this countries. In this meaning, a study economic variation in Germany, U.S. and Hong Kong as benchmark economies, around the economies of Honduras and Costa Rica, to show the pivot points, the similarity of the oscillations and trends, periods, ranges and offsets, deviations and asymmetries that can exist between these economies to create a thoughtful analysis of the trends. For this; was considerate like basis, the variations in Gross Domestic Product (GDP), GDP per capita, cash capital profit or deficit, monetary reserves, external debt and foreign direct investment, for a period of nineteen years.
\end{abstract}

Keywords: Economics Cycle, Globalization, Economic Asymmetry, Economies Dependence. 
Revista Económica y Administración (E\&A), vol 4(2), pp. 103-122, @ 2013, IIES-UNAH Oscilaciones Macroeconómicas de Honduras y Costa Rica en Relación a las Economías de los Países Desarrollados

\section{INTRODUCCIÓN}

A través del tiempo, las economías han presentado oscilaciones cuyos periodos han sido marcados por épocas de recesiones, depresión, crecimiento y expansión, con patrones recurrentes y sistemáticos vinculadas a variables de amplitud y duración, las cuales en los países desarrollados, han sido ampliamente analizados, de forma que les ha posibilitado a entendidos en la materia, medir su volatilidad ante cualquier evento, ya sea de tipo político, económico y social, permitiendo a los gobiernos hacer visible la información y sus cambios para gestar tomas de decisiones acertadas en materia micro y macro económica.

Sin embargo, a pesar que las principales naciones cuentan con todos los mecanismos e insumos para prever, monitorear y contrarrestar los cambios abruptos en la economía, no han logrado determinar aspectos fundamentales que les accedan una identificación de forma preventiva, sino totalmente correctiva, fomentando nuevas y rigurosas políticas económicas que mantengan sus indicadores y estabilicen su dinámica comercial, impactando directamente en las economías dependientes a dichos países, las cuales giran sus indicadores económicos al ritmo de las potencias mundiales, evidenciando oscilaciones de crecimiento o decrecimiento a través del tiempo en total comparsa con la economía a la cual depende.

Para identificar dichas relaciones de dependencia y construir un análisis holístico de las similitudes de comportamiento de los principales indicadores macroeconómicos, se lleva a cabo una reflexión de los cambios económicos de los países desarrollados en comparación a los países en vías de desarrollo, fundamentado en el análisis de las potencias por regiones (Por Norte América se razonó: Estados Unidos, por Europa: Alemania, por Asia: Republica Administrativa del Hong Kong, y por Centro América: Honduras y Costa Rica), para visualizar todas las oscilaciones y relaciones de dependencia existente entre economías, centrándonos en Honduras y Costa Rica, la fuente del análisis lo compone las variaciones en el Producto Interno Bruto para un periodo de tiempo de diezy nueve años.

El estudio se refuerza con el análisis complementario del Producto Interno Bruto Per cápita, Superávit o déficit de efectivo, Deuda externa acumulada y la Inversión extranjera directa. Las variaciones de los indicadores enunciados, indicarán con qué similitud sufren alteraciones las economías en estudio y evidenciara las posibles dependencias entre sí. Lo citado brinda la posibilidad de esquematizar 
OSCILACIONES MACROECONÓMICAS DE HONDURAS Y COSTA RICA EN RELACIÓN A LAS ECONOMÍAS DE LOS PAÍSES DESARROLLADOS

Vol. 4

E\&A

IIES

106

propuestas que inviten al análisis reflexivo y adopción de modelos económicos certeros para alcanzar oscilaciones económicas controladas.

\section{CICLOS ECONÓMICOS}

Los ciclos se pueden analizar desde diversos enfoques, desde aquellos que consideran que los ciclos u oscilaciones económicas son variaciones específicas de las economías basadas en la moneda y su actividad comercial, en donde el aparato productivo se encuentra organizado por empresas que buscan optimizar sus recursos y maximizar sus ganancias, hasta aquellos enfoques fundamentados en la teoría Keynesiana (Hyman P. Minsky, 1987). Dicha conceptualización derivada de los trabajos de Burns y Mitchell en el National Bureau of Economic Research (NBER), se extraen inmediatamente dos características básicas en el estudio del ciclo económico: la variabilidad en la evolución del ciclo y la existencia de fluctuaciones en un amplio conjunto de indicadores o comovimientos. Esta última característica es la que resulta más interesante desde el punto de vista de la Teoría Económica, existiendo un amplio número de investigaciones sobre la caracterización y explicación de las fluctuaciones entre variables. Una revisión parcial de los mismos desde una perspectiva histórica puede consultarse en Zarnowitz (1991), Zarnowitz (1996) ó Kydlan (1995) y (Carcedo, 2002).

Los ciclos económicos cuentan con cuatro fases: 1. Auge, 2. Recesión, 3. Depresión, 4. Recuperación / Reactivación. El auge es el punto más elevado donde se alcanza una rigidez que da paso al inicio de una recesión, etapa considerada como la fase descendente del ciclo, caracterizada por la caída de la inversión, producción y el empleo. La depresión es el punto más bajo del ciclo, representado por un alto nivel de desempleo y un bajo nivel de demanda por parte de los consumidores en relación a la capacidad productiva de bienes. Por su parte, la reactivación, es la renovación de capital cuyo efectos multiplicadores impactan en la economía del país generando una etapa plena de expansión y crecimiento (Andrew J. Filardo, 2012).

Asimismo, los ciclos económicos cuentan con diferentes clases determinadas por su duración en el tiempo. Si se presenta en un periodo menor a 40 meses se considera un ciclo económico corto. Sin embargo, si su duración va más allá de los 40 meses y no sobre pasa los 8 años se considera como crisis cíclicas o de Juglar. A su vez, los ciclos denominados largos son aquellos cuya duración ronda los 50 a 60 
Revista Económica y Administración (E\&A), vol 4(2), pp. 103-122, @ 2013, IIES-UNAH Oscilaciones Macroeconómicas de Honduras y Costa Rica en Relación a las Economías de los Países Desarrollados

años, los cuales de manifestarse durante la etapa de expansión, el crecimiento y los ascensos son prolongados. (Clément Juglar, 1862).

\section{OSCILACIONES ECONOMICAS EN AMÉRICA LATINA}

Contextualizando la teoría a los acontecimientos que se antecedió, en la última década la economía mundial ha sufrido una desaceleración característica producto de los cambios en las dinámicas comerciales de las potencias (Estados Unidos, Alemania, Francia, Italia, Japón) la cual trajo consigo una serie de crisis económicas mundiales (BCIE, 2010). A partir del 2007, dichas crisis se agudizaron, trasladándose al sector real de las economías como consecuencia de la iliquidez en las economías familiares, decrecimiento de la demanda y aumentando la privación de los créditos. (Alexander Bobróvnikov, 2002).

La contracción global de la economía condujo a los gobiernos y a las entidades que regulan las reservas, a insertar más flujo de efectivo en el mercado. Además, se buscó disminuir las tasa de interés, disminuir el gasto público y como complemento a estas acciones, el monitoreo constante de las variaciones y posibles riesgos que impidieran la estabilización y crecimiento de la economía (BCIE, 2010). Sin embargo, los resultados de estas medidas no obtuvieron el impacto deseado, el crecimiento fue lento, alcanzando un promedio un 3\% para el año 2010. El efecto directo de los excesos previos se reflejaron en las tasas elevadas de desempleo en los países desarrollados, el mercado de la vivienda deprimido, las presiones en las economías por la continua inflación y los cambios en las políticas monetarias, que en combinación con las dificultades fiscales, aumento del gasto público y presiones financieras, incidieron directamente en la curva de crecimiento de la economía mundial (Fondo Monetario Internacional, 2011).

Como contraparte a lo suscitado, las economías emergentes han tenido un desempeño más sólido y constante sustentado en la elevada captación de capitales producto de las fugas de efectivo en las grandes economías. Se estimó que estas economías alcanzaron un crecimiento del $7.25 \%$ en promedio para el año 2010 (Fondo Monetario Internacional, 2011) descansando el mismo en su base fiscal y financiera bien fundamentada, así como la reactivación de la exportación y una demanda interna inducida por las políticas monetarias y fiscales emprendidas (Alexander Bobróvnikov, 2002). Focalizando lo expuesto a los países de América Latina y el Caribe las repercusiones fueron directamente en el ámbito comercial, oscilaciones a nivel intra-latinoaméricano alcanzaron un crecimiento bajo pero 
OSCILACIONES MACROECONÓMICAS DE HONDURAS Y COSTA RICA EN RELACIÓN A LAS ECONOMÍAS DE LOS PAÍSES DESARROLLADOS

Vol. 4

E\&A

IIES

108

significativo (1.4\%), incidiendo directamente en las desaceleraciones económicas pronunciadas en la región que se vieron diferenciadas en los países productores de hidrocarburos y los países de Centroamérica y el Caribe (paso de un 23\% en 2011 a una estimación del 1.6\% para el año 2012, (CEPAL Naciones Unidas, 2012)). Las variaciones en las economías, dieron como resultado una reducción en el superávit de la cuenta comercial de bienes en la balanza de pagos, la cual paso de $1.4 \%$ en 2011 a 1.0\% para 2012 en relación al PIB.

A pesar de las dificultades en la economía mundial, las economías de américa latina mostraron resistencia para afrontar los golpes económicos externos, impactándoles pero no arreciando como en las economías de primer mundo. Se registró un crecimiento del PIB de la región del $0.9 \%$ por encima del crecimiento del PIB mundial (2.2\%). En cuanto al desempleo se mostró una mejoría al pasar de una tasa del $6.9 \%$ en 2011 a $6.4 \%$ para el año 2012, disparando la tasa de ocupación en cada uno de los países de la región.

\section{ANÁLISIS DE LAS OSCILACIONES DE LAS ECONOMÍAS OBJETO DE ESTUDIO}

En este apartado se contempla el estudio de los ciclos económicos de las potencias para desarrollar un análisis comparativo de las economías consideradas más fuertes del mundo (Estados Unidos, Alemania y Hong Kong) para visualizar el grado de dependencia económica de los países en vías de desarrollo de Centroamérica, puntualizados en Costa Rica (por ser un país punta de lanza en la región) y Honduras (por ser el país que da origen a esta investigación). El análisis comparativo se construyó a partir de tres segmentos de estudio, el primero de ello, por medio de las variaciones del PIB, Ahorros, Balanza de Pagos, y Reserva total. El segundo, en función de las variaciones de los compromisos adquiridos. El tercer segmento, centrado en el ingreso percibido en las economías producto de la atracción de la inversión extranjera directa.

\section{A) Primer Segmento de análisis: Variaciones del PIB en el tiempo (1993- 2012)}

El primer bloque de análisis consiste en el estudio de las variaciones del Producto Interno Bruto (PIB) de las economías de Alemania, Estados Unidos, Hong Kong, Honduras y Costa Rica, para determinar los rangos, la curtosis, la varianza, la asimetría, la dependencia, los puntos de giros, las fases de los ciclos, y sus duraciones. 
1. Crecimiento del PIB (\% anual): Tasa de crecimiento anual porcentual del PIB a precios de mercado en moneda local.

Tabla 1. Estadísticas de Crecimiento del PIB en \%.

\begin{tabular}{|crrrrrrrrrrr} 
Crecimiento del PIB (\% anual) & 1993 & 1994 & 1995 & 1996 & 1997 & 1998 & 1999 & 2000 & 2001 & 2002 \\
\hline Alemania & -1.00 & 2.50 & 1.70 & 0.80 & 1.70 & 1.90 & 1.90 & 3.10 & 1.50 & 0 \\
\hline Estados Unidos & 2.90 & 4.10 & 2.50 & 3.80 & 4.50 & 4.40 & 4.90 & 4.20 & 1.10 & 1.8 \\
\hline Hong Kong & 6.20 & 6.00 & 2.40 & 4.30 & 5.10 & -5.90 & 2.50 & 7.70 & 0.60 & 1.70 \\
\hline Honduras & 6.20 & -1.30 & 4.10 & 3.60 & 5.00 & 2.90 & -1.90 & 5.70 & 2.70 & 3.8 \\
\hline Costa Rica & 7.40 & 4.70 & 3.90 & 0.90 & 5.60 & 8.40 & 8.20 & 1.80 & 1.10 & 2.9 \\
\hline & & & & & & & & & & \\
\hline Crecimiento del PIB (\% anual) & 2003 & 2004 & 2005 & 2006 & 2007 & 2008 & 2009 & 2010 & 2011 & 2012 \\
\hline Alemania & -0.4 & 1.2 & 0.7 & 3.7 & 3.3 & 1.1 & -5.1 & 3.7 & 3 & - \\
\hline Estados Unidos & 2.6 & 3.5 & 3.1 & 2.7 & 1.9 & -0.4 & -3.5 & 3 & 1.7 & - \\
\hline Hong Kong & 3.10 & 8.70 & 7.40 & 7.00 & 6.50 & 2.10 & -2.50 & 6.80 & 4.90 & - \\
\hline Honduras & 4.5 & 6.2 & 6.1 & 6.6 & 6.2 & 4.2 & -2.1 & 2.8 & 3.4 & - \\
\hline Costa Rica & 6.4 & 4.3 & 5.9 & 8.8 & 7.9 & 2.7 & -1 & 4.7 & 4.2 & -
\end{tabular}

Fuente. Indicadores de Política económica y deuda del Banco Mundial.

En los datos presentados en la tabla 1, se observa que países como Alemania y Estados Unidos tienen ciclos económicos de tipo pro-cíclicos, cuya aceleración es más estable y prolongada, constando que las desaceleraciones son más cortas y menos pronunciadas, a excepción del desplome del año 2009.

\section{Tabla 2. Análisis estadístico de los datos del indicador de Crecimiento del PIB}

\begin{tabular}{lcccccc}
\multicolumn{6}{c}{ Pais / Estadistica } & Alemania Estados Unidos Hong Kong Honduras Costa Rica \\
\hline \hline Minimos & -5.10 & 3.50 & 5.90 & -2.10 & - \\
Maximos & 3.70 & 4.90 & 8.70 & 6.60 & 8.80 \\
Media & 1.33 & 2.57 & 3.93 & 3.62 & 4.67 \\
Mediana & 1.70 & 2.90 & 4.90 & 4.10 & 4.70 \\
Desv estandar & 2.05 & 1.97 & 3.71 & 2.71 & 2.81 \\
coeficiente de var & 1.54 & 0.77 & 0.94 & 0.75 & 0.60 \\
Varianza & 4.20 & 3.89 & 13.76 & 7.34 & 7.89 \\
Curtosis & 4.56 & 4.15 & 1.48 & 0.54 & - & 0.69 \\
Coeficiente de asimetria & 0.65 & 1.01 & -1.12 & -1.10 & - & 0.59 \\
Coeficiente de asimetria de pearson & 0.32 & 0.22 & 0.50 & 1.00 & 0.28 \\
Pronostico para 2013 & $1.16 \%$ & $0.290 \%$ & $3.54 \%$ & $3.14 \%$ & $3.17 \%$
\end{tabular}

Fuente: Construcción propia.

La revisión del análisis estadístico (Tabla 2) nos permite determinar que la economía que ha presentado los mínimos de crecimiento más elevados es Hong Kong (-5.90\%) seguido de Alemania (-5.10), Estados Unidos (3.5\%). Por su parte, las economías que alcanzaron los niveles más altos de crecimiento fueron Hong Kong ( $8.70 \%$ en 2004$)$ y Costa Rica ( $8.80 \%$ en 2006). Los países con menor nivel 
OSCILACIONES MACROECONÓMICAS DE HONDURAS Y COSTA RICA EN RELACIÓN A LAS ECONOMÍAS DE LOS PAÍSES DESARROLLADOS

Vol. 4 $\mathrm{N}^{\circ} 2$ 2013

E\&A

IIES

110

de crecimiento, son Alemania (3.70\% en 2010), Estados Unidos (4.90\% en 1999) y Honduras (6.60\% en 2006), salvaguardando las diferencias de montos del PIB de cada país.

De igual forma se aprecia que la economía con mayor desviación estándar es Hong Kong $(3.71 \%)$ por su condición de mercado emergente, seguido de Costa Rica (2.81\%), Honduras (2.71\%), Alemania (2.05\%) y Estados Unidos (1.97\%). En cuanto a la proporción de la varianza a partir de la combinación de datos extremos respecto a la media y en contraposición de aquellos datos alejados de la misma, economías como las de Alemania (4.56) y Estados Unidos (4.15) presentan una mayor concentración de datos de crecimientos del PIB muy cercanos a la media. Por otro lado, economías como la de Hong Kong (1.48), Honduras (0.54) y Costa Rica (-0.59) tienen mayores variaciones respecto a la media por su condición de economías emergentes.

A continuación se presentan de forma gráfica las variaciones de las economías solidas objeto de estudio.

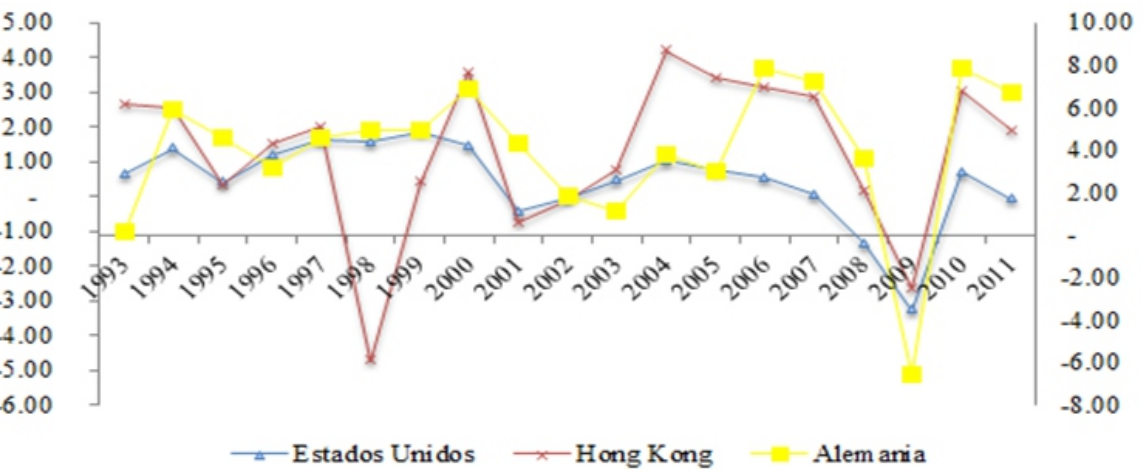

Fig. 1. Ciclo Económico de las principales potencias mundiales, periodo del 1993 al 2011

Fuente: Indicadores de Política económica y deuda del Banco Mundial.

Del análisis de la figura 1, se determina que para el periodo 1993 al 2011 Alemania tuvo cuatro puntos de giro, marcando cuatro ciclos económicos diferentes, para el año 1993 oscilo de $-1 \%$ a $2.5 \%$ en el año 1994. La segunda cima se obtuvo en el año 2000 alcanzando un $3.10 \%$ de crecimiento del PIB y llegando a $-0.4 \%$ en 2003. El tercer punto de giro ocurrió en 2003 de $-0.4 \%$ hasta $3.7 \%$ en 2006 , punto 
Revista Económica y Administración (E\&A), vol 4(2), pp. 103-122, @ 2013, IIES-UNAH Oscilaciones Macroeconómicas de Honduras y Costa Rica en Relación a las Economías de los Países Desarrollados

donde empezó la desaceleración de su economía hasta llegar al nuevo punto de giro en 2009 (-5.1\% de crecimiento del PIB). Para este último punto de giro la economía alemana presenta un repunte de $3.7 \%$ en 2010 y 3\% 2011.

Por su parte, la economía de Estados Unidos para el periodo de 1993 a 2011 tuvo tres puntos de giro. El primer punto de giro ocurrió en el año 1995, de $0.10 \%$ paso $2.50 \%$ de crecimiento del PIB. A partir de 1996 la economía presento un crecimiento hasta el año 1999 (4.90\% de crecimiento del PIB). Del año 2001 hasta el 2004 la economía empezó a ascender hasta alcanzar un crecimiento máximo de $3.5 \%$, en 2005 inicia la desaceleración de la economía hasta caer a valor negativo del PIB (-3.5\% en 2009). Después de este último punto de giro la economía de Estados Unidos se dinamizó, no obstante solo pudo crecer en 3\% para el 2010, cayendo a $1.7 \%$ su PIB en el año 2011.

Continuando el análisis por economía, Hong Kong ha tenido cuatro puntos significativos de giro. El primero de ellos, cuando su PIB cayó de 6\% a 2.40\% en 1995. Los años de 1996 y 1997 significaron un crecimiento de $4.30 \%$ y 5.10\% respectivamente hasta alcanzar el segundo punto de giro en 1998 con una desaceleración del PIB del $-5.90 \%$. Después de este giro la economía experimento un crecimiento de $2.50 \%$ en 1999 y $7.7 \%$ en 2000 en su PIB, a pesar de ello la economía de Hong Kong se desplomó en 2001 con un crecimiento de $0.60 \%$. El crecimiento del PIB empezó de nuevo en el año 2002 (1.70\%) hasta llegar a su punto máximo en tres años, donde su crecimiento fue de $8.70 \%$, cuatro años después ocurrió una desaceleración que alcanzó su punto mínimo de $-2.50 \%$ en 2009 , de ello se determina que las economías solidas tienen ciclos con duraciones promedios de 3 a 7 años, con distancias entre cimas de 4 a 5 años, a excepción de las economías asiáticas por su característica emergente.

Por su parte, las oscilaciones en las economías de los países en vías de desarrollo han sufrido las mismas tendencias de las grandes potencias, por la directa e indirecta dependencia económica existente. En este sentido, se presenta la figura 3. Que muestran las similitudes en las oscilaciones del crecimiento del PIB de Honduras y Costa Rica en relación a Estados Unidos. 
Vol. 4 $\mathrm{N}^{\circ} 2$ 2013

E\&A

IIES

112
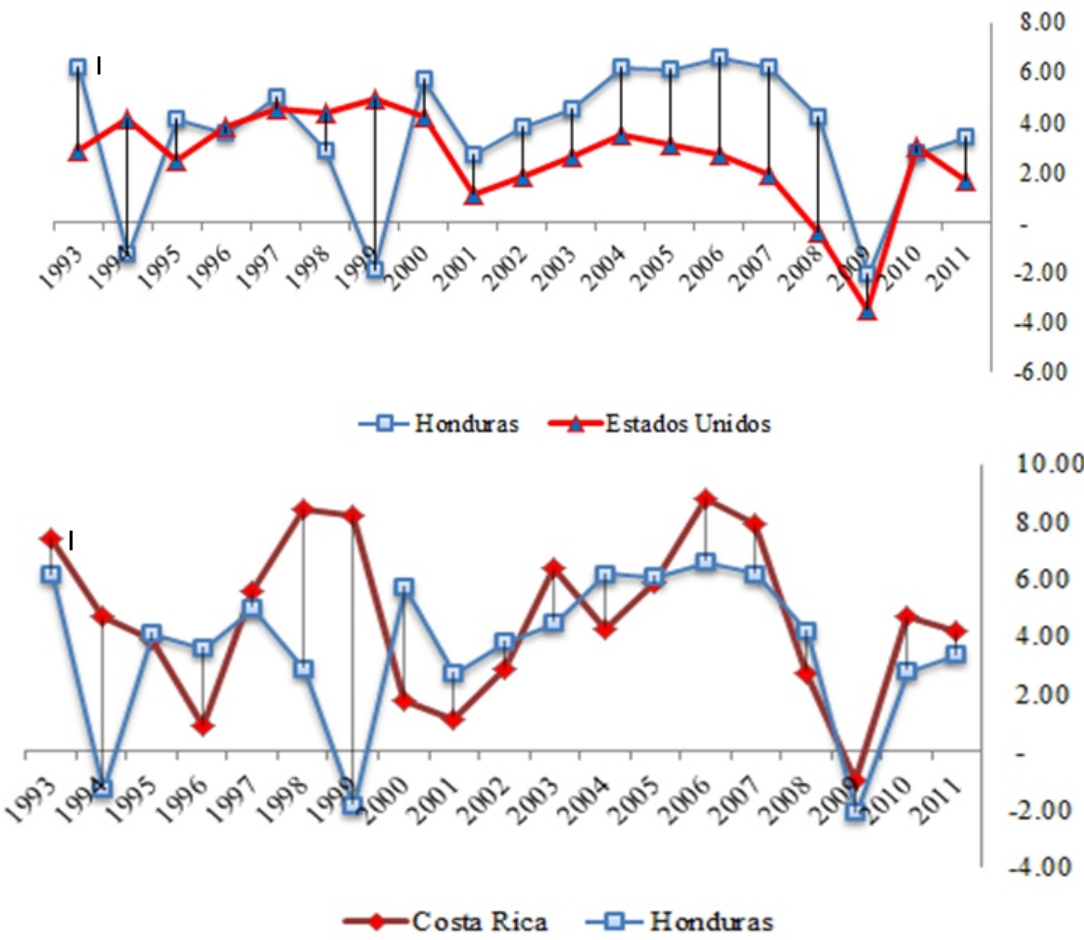

\section{Fig. 2. Análisis gráfico del ciclo económico de Honduras, EEUU y Costa Rica, periodo del 1993 al 2011}

Fuente: Indicadores de Política económica y deuda del Banco Mundial.

La figura 2, presenta las oscilaciones en la economía de Honduras, marcada por cuatro cambios, el primer punto de giro del ciclo de crecimiento del PIB fue en el año 1994 cuando su economía se desplomó con una desaceleración de -7.50\% (E1 crecimiento para 1994 fue -1.30\%). A partir de 1995 el PIB empieza a ascender hasta alcanzar su posición máxima en 1997 con un crecimiento del 5\%. No obstante, dos años bastaron para que cayera a $-1.90 \%$. La duración de este ciclo fue de cuatro años. Para el año 2000 se presentó un repunte en PIB de 5.70\% no sostenible, resultando en un nuevo punto de giro ante la caída del mismo a $2.70 \%$. Ante ello, en el año 2002 se inicia un nuevo ciclo que llego a su cima en el año 2006 con un crecimiento de $6.60 \%$ del PIB, punto donde inicia la caída del ciclo hasta alcanzar una desaceleración de $-6.3 \%$ en 2009 , significando el cuarto punto de giro. La duración del ciclo: siete años. Para los años 2010 y 2011 la economía de Honduras empieza a oscilar de manera positiva. 
En la figura 3, se aprecia un gráfico comparativo de las tendencias del PIB de Honduras en comparación con el de Costa Rica, en el mismo, se aprecia que sus puntos de crecimiento solo se acercan en los años: 1995, 1997, 2002, 2005, 2008 y 2011, el resto de los puntos son inversamente proporcionales, esto atribuido al origen de sus socios comerciales estratégicos. Costa Rica presenta tres puntos de giro durante el periodo de 1993 a 2011. El primero de ellos, en el año de 1996 con un $0.90 \%$ de crecimiento del PIB, dicho ascenso se mantuvo por dos años hasta llegar en el año de 1998 a un $8.40 \%$, dos años más tardes dio lugar el siguiente punto de giro, marcado por una desaceleración del $-7.30 \%$ en relación a su valor máximo. El ciclo tuvo una duración de cuatro años.

El PIB de Costa Rica continuo con un ascenso progresivo con un promedio de crecimiento de $2.667 \%$ por año, hasta alcanzar su nivel máximo de $8.8 \%$ en el año 2006. Dos años más tarde, el comercio de Costa Rica se desplomó hasta obtener para el año 2009 un nivel del PIB de -1\%, originando un giro en su ciclo, duración: siete años. A su vez, para los años 2010 y 2011 Costa Rica muestra una recuperación de su crecimiento económico (4.7\% v 4.2\%).
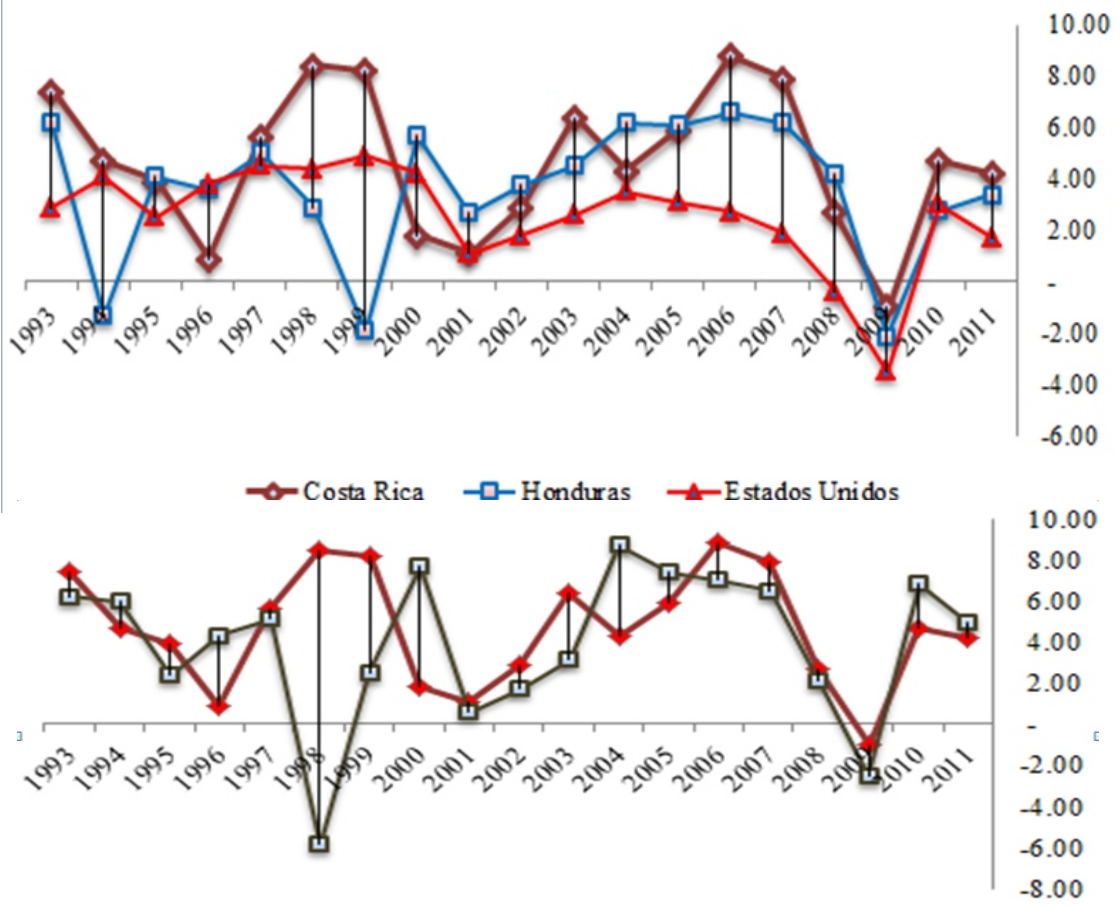

E\&A

IIES

113

\section{Fig. 3. Ondas de expansión y contracción del PIB de EEUU, Hong Kong, Honduras y Costa Rica, periodo del 1993 al 2011}

Fuente: Datos de los indicadores de Política económica y deuda del Banco Mundial. 
OSCILACIONES MACROECONÓMICAS DE HONDURAS Y COSTA RICA EN RELACIÓN A LAS ECONOMÍAS DE LOS PAÍSES DESARROLLADOS

Vol. 4 $\mathrm{N}^{\circ} 2$ 2013

E\&A

IIES

114

1. Superávit/déficit de efectivo (\% del PIB): El superávit/déficit de efectivo es el ingreso (incluso por donaciones) menos el gasto, menos la adquisición neta de activos no financieros.

\section{Tabla 3. Estadísticas del Superávit/Déficit de Efectivo durante el periodo comprendido de 1993 a 2012}

\begin{tabular}{|c|c|c|c|c|c|c|c|c|c|c|}
\hline Superávit/déficit de efectivo (\% del PIB) & 1993 & 1994 & 1995 & 1996 & 1997 & 1998 & 1999 & 2000 & 2001 & 2002 \\
\hline Alemania & - & - & -8.10 & -2.20 & -1.50 & -1.70 & -1.30 & 1.40 & -1.50 & -2.00 \\
\hline Estados Unidos & - & - & - & - & - & - & - & 0.50 & -2.60 & -2.60 \\
\hline Hong Kong & . & . & . & . & . & . & . & . & . & .6 .80 \\
\hline Honduras & - & - & - & - & - & - & - & - & - & - \\
\hline Costa Rica & - & . & - & - & - & . & . & - & - & - \\
\hline Superávit/déficit de efectivo (\% del PIB) & 2003 & 2004 & 2005 & 2006 & 2007 & 2008 & 2009 & 2010 & 2011 & 2012 \\
\hline Alemania & -2.20 & -2.40 & -2.30 & -1.30 & -0.30 & -0.30 & -2.20 & -3.10 & - & - \\
\hline Estados Unidos & -3.80 & .3 .60 & .2 .80 & $\cdot 1.80$ & .2 .20 & -5.30 & -10.40 & -10.10 & - & - \\
\hline Hong Kong & -5.90 & -1.00 & 1.40 & 3.90 & 6.70 & 0.60 & 1.10 & 4.20 & - & - \\
\hline Honduras & -3.00 & -0.70 & .0 .80 & 0.90 & -1.10 & -0.20 & .4 .60 & -3.10 & - & . \\
\hline Costa Rica & . & . & - & - & . & -0.80 & $=3.40$ & .2 .80 & . & . \\
\hline
\end{tabular}

Fuente. Indicadores de Política económica y deuda del Banco Mundial

Del análisis de la tabla 3, se obtiene que para el año 1995, Alemania presentaba un déficit de -8.10, durante el periodo de 1995 a 1999 la balanza de pagos disminuyo su déficit alcanzando un valor de -1.30. Su mejor año fue el 2000 cuando alcanzó un superávit de 1.40 en relación al PIB. A partir del 2001, la balanza de pagos empezó a mostrar signos de debilitamiento, en 2004 (-2.40) y en 2010 (-3.10). En cuanto a Estados Unidos se refiere, la balanza de pagos ha venido en deceso durante el último quinquenio, mostrando signos de recuperación en los años 2005 a 2007. Los siguientes años mostró una caída significativa que alcanzó un déficit de -10.10 para 2010. Por su parte, la economía de Hong Kong, presentó un déficit de -6.80 en 2002, sin embargo los años subsiguientes ha sabido elevar su ventaja competitiva e incrementar sus inversiones y exportaciones, reflejadas en su superávit de 1.40 en 2005 hasta alcanzar 6.70 en 2007. Manteniéndose variable a través de los siguientes tres años (0.60 en 2008; 1.10 en 2009, 4.20 en 2010).

En el caso de Honduras, en los años 2003 (-3.0), 2009 (-4.60) y 2010 (-3.10) se presentaron los mayores déficits en su balanza de pago. Dicho ciclo rondo cinco años, de 2004 a 2008, con una tendencia hacia un superávit mínimo pero significativo para una economía subdesarrollada. Al realizar el análisis anual se aprecia que el mejor año para la balanza de pagos y para el país fue el 2006, se alcanzó un superávit de 0.9. Sin embargo, los sucesos políticos dinamitaron la 
economía y comenzó el desplome ante el incremento del gasto público, para el año 2009 el déficit fue de -4.6 y en 2010 de -3.1.

Por otro lado, Costa Rica solo presentaba datos para el periodo del año 2008 al 2010. Por lo tanto, se denota que su periodo más alto fue en el año de 2009 donde alcanzó un déficit de -3.4. No obstante, la continua caída de este indicador estuvo presente el siguiente año con un déficit de -2.8. La dependencia hacia las grandes economías, el incremento del gasto público del estado como sustentador de una sociedad que crece a mayor ritmo que la economía misma. El elemento diferenciador de este análisis se centra en las variaciones de los países emergentes quienes a través de sus políticas de desarrollo han alcanzado un crecimiento sostenido.

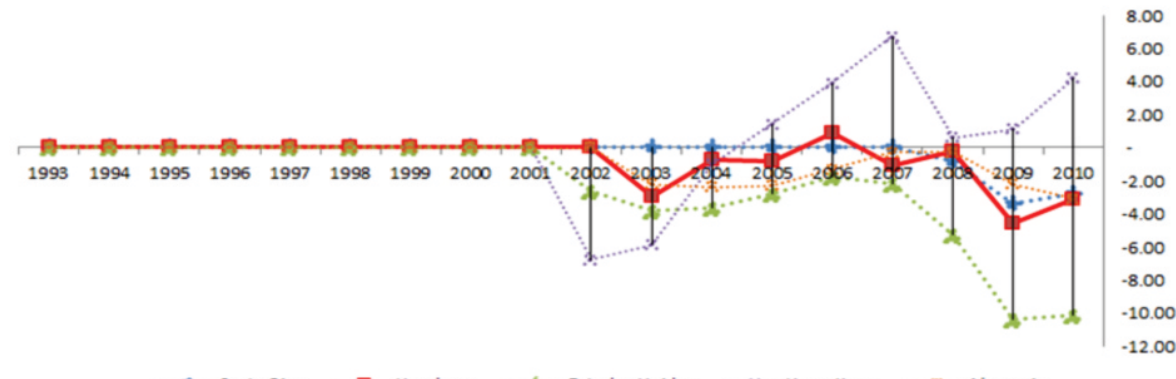

E\&A

IIES

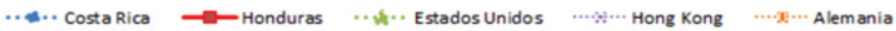

Fig. 4. Superávit/ déficit de efectivo \% en relación al PIB para Alemania, EEUU, Hong Kong, Honduras y Costa Rica, periodo del 1993 al 2011

Fuente: Datos de los indicadores de Política económica y deuda del Banco Mundial.

B) Segundo segmento de análisis: Variaciones de los compromisos contraídos por las naciones como factor de análisis de los ciclos económico

El segundo segmento de este análisis comprende las variaciones en los compromisos contraídos por los países en estudio ${ }^{1}$. La importancia de este análisis es identificar como el grado de endeudamiento bajo una utilización efectiva puede contribuir al enriquecimiento de una nación.

1. Deuda externa acumulada, total (DOD, US\$ a precios actuales): La deuda externa total es el monto adeudado a los no residentes, que se rembolsa en divisas, bienes o servicios. 
OSCILACIONES MACROECONÓMICAS DE HONDURAS Y COSTA RICA EN RELACIÓN A LAS ECONOMÍAS DE LOS PAÍSES DESARROLLADOS

\section{Tabla 4. Estadísticas de la Deuda externa acumulada total a precios actuales en decimos de millones.}

Vol. 4 $\mathrm{N}^{\circ} 2$ 2013

E\&A IIES
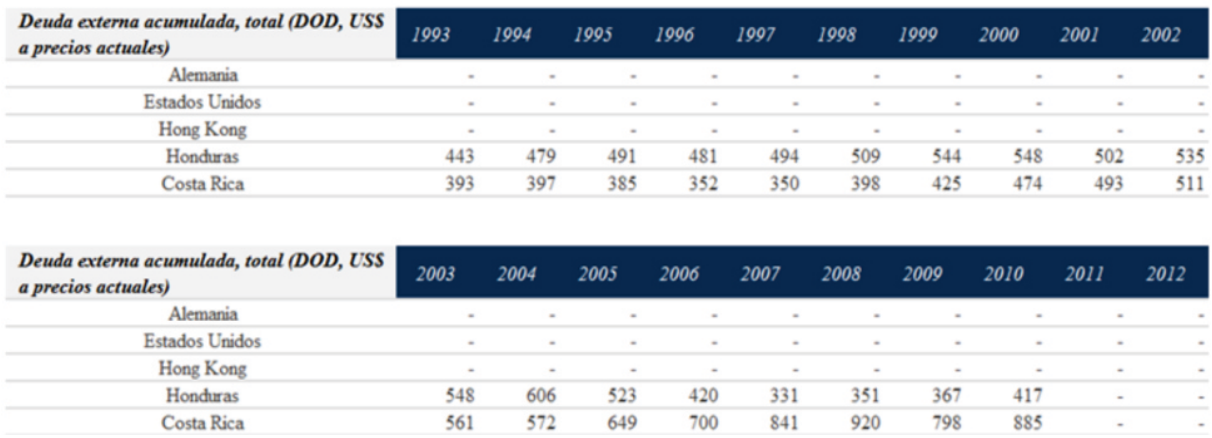

Fuente. Indicadores de Política económica y deuda del Banco Mundial al 2012.

Los datos de la tabla 4, dan paso a sustentar que países como Honduras dependen del endeudamiento para su subsistencia y por ende la dependencia directa de sus economías hacia los países prestatarios de los recursos. Ante ello, Honduras presenta una curva ascendente de endeudamiento, del año 1994 al 2000 las tasas de crecimiento de la deuda rondaban un promedio de $3 \%$ anual, a pesar de ello, en el año de 2001 cayó a - $8 \%$, siendo el primer ciclo para las variaciones de la deuda.

Del año 2002 al 2004 la deuda se incrementó a una tasa promedio anual del 7\%, alcanzando su máximo valor (11\%) en 2004. A partir del 2005 hasta el 2007 los porcentajes adeudados disminuyeron a una tasa promedio del $18 \%$ anual, como resultado de la condonación de deudas y otras estrategias. A partir del año 2008 hasta el 2010 el país se endeudo a una tasa promedio del 8\% anual. En el caso de Costa Rica, a través de la historia muestra niveles de Endeudamiento mayores a los que posee Honduras, con la diferencia que la utilización de los mismos se ha visto en ganancias redituables para el estado y la sociedad.

Desde 1994 hasta el año 2010, Costa Rica se ha endeudado a un promedio anual del 5\%, siendo sus picos los años 2005 (14\%), 2007 (20\%), y 2010 (11\%). Del análisis se observa que solo en cuatro años Costa Rica disminuyo su tasa de endeudamiento (1995 (-3\%), $1996(-8 \%), 1997(-1 \%)$ y $2009(-13 \%))$. A continuación se presenta de forma gráfica las tendencias expuestas en los párrafos anteriores. 
Fig. 5. Deuda Externa Acumulada, variaciones a través del ciclo económico como un análisis comparativo, periodo del 1993 al 2011

Fuente: Datos de los indicadores de Política económica y deuda del Banco Mundial.

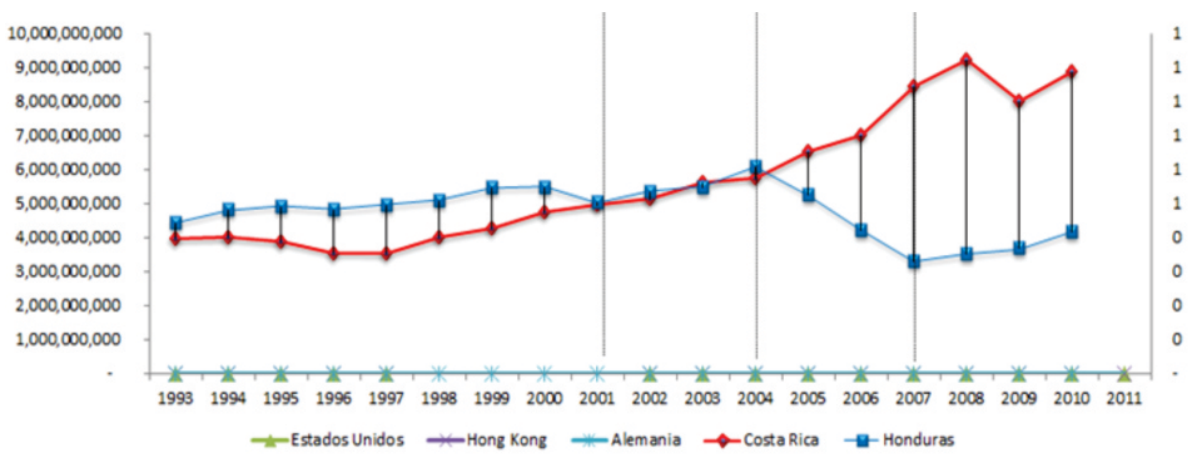

C) Tercer segmento de análisis: Variaciones de los ingresos externos en los ciclos económicos

Los ingresos externos son de gran ayuda para las naciones cuando sus economías necesitan capital para poder generar la inversión, empleo, mejora en la calidad de vida, incremento de sus exportaciones y saldos positivos en sus balanzas de pagos.

1. Inversión extranjera directa, entrada neta de capital (balanza de pagos, US\$ a precios actuales): La inversión extranjera directa constituye la entrada neta de inversiones para obtener un control de gestión duradero de una empresa que funciona en un país que no es el del inversionista.

Si bien es cierto, las economías potencias objeto de estudio serán las que resulten más atrayentes para las Inversiones Extranjeras Directas (IED), infraestructura, escenario y clima de negocios que presenta, es importante hacer la relación de la fuga de capitales de las potencias hacia los países emergentes por las variables básicas como ser puntos estratégicos de logística, y abaratamiento de la mano de obra, así como las concesiones otorgadas por las naciones. En este sentido, se aprecia que en los Estados Unidos, a partir del año 1995 al 2000 sus recursos por este concepto crecieron alcanzando un valor de USD 321274000,000. A pesar de ello, a partir del año 2001 se desplomó la IED hasta caer a su punto más bajo en 2003 (USD 63750000,000), En el año 2008 se recuperó la IED (USD $310093000,000)$ sin ser la misma sostenible, colapsando por los cambios en la economía mundial, los años siguientes presentaron un descenso que finalizó con USD227865000, 000 en 2011. 
OSCILACIONES MACROECONÓMICAS DE HONDURAS Y COSTA RICA EN RELACIÓN A LAS ECONOMÍAS DE LOS PAÍSES DESARROLLADOS

Vol. 4

E\&A

IIES

118

Por su parte, Alemania para el primer periodo de análisis muestra un comportamiento oscilatorio similar al de la IED de Estados Unidos, con un desfase de dos años. Es a partir del año 1997 donde culmina su valle llegando a su punto máximo en el año 2000 (USD 210085430,608), punto donde la IED Alemana empieza su caída, hasta alcanzar el valor mínimo de USD -9802914,900 en 2004. Después de la estrepitosa caída, la IED tuvo un comportamiento de ascenso de tres años hasta alcanzar nuevamente su punto más alto en 2007 (USD 80592535,711), a partir del año 2008 y 2009 la IED tuvo un descenso significativo que lo llevo a un valor de USD 8454528,204. Por otra parte, la IED en la economía de Hong Kong gesta su primer periodo en 1998, con una duración promedio de seis años, siendo el año de 2003 su punto de giro. El valor máximo alcanzado en este periodo fue de USD 61923897,912 en el año 2000. Del año 2004 hasta el año 2011 la IED ha mostrado una tendencia de ascenso continuo a pesar de su debilitamiento en el año 2009 (USD 52395154,100).

En el caso de Honduras y Costa Rica, la IED ha presentado un comportamiento en ascenso a través del periodo de estudio (1993 a 2011). Tal es el caso de Honduras, la cual a partir del año 1994 hasta el año 2000, mostró un ascenso continúo que representó una suma de USD 381659,149 para el 2000, ver figura 6. En 2001, la IED tuvo una leve caída a USD 304242,923 y en 2002 alcanzó USD 275167,835. No obstante, logró retomar el crecimiento continuo durante cinco años consecutivos hasta 2008 (USD 929308,627) punto donde comienza de nueva caída. El clima de inseguridad que vive Honduras a partir de los sucesos políticos, más las dificultades en materia de procesamiento e instalación de una empresa, así como los trámites para participar como inversionistas han mermado el crecimiento de la IED en el país.

A diferencia de Honduras, el sistema político y jurídico de Costa Rica ha permitido instaurar un clima de inversión totalmente favorable para atraer la IED. Del año 1994 a 1999 Costa Rica sufrió un repunte significativo en su IED que permitió se generaran USD 619469,212. Para el 2001, la economía costarricense tuvo una desaceleración en su IED, producto de la caída de la economía bursátil, en donde los componentes electrónicos y de procesamiento se vinieron abajo. A partir del año 2002 la IED mostró una tendencia hacia el ascenso continuo hasta llegar a su punto máximo en el año 2008 (USD 2078238,732). Los años 2009 y 2010, significaron una caída en la IED recibida en Costa Rica aunque no tan significativa en relación a su crecimiento. Para el año 2011 los valores de la IED de Costa Rica mostraron un repunte sustancial alcanzando un total de USD 2104114,969 dando solidez e impulso al comercio y su economía. 
Fig. 6. Cambios en la Inversión Extranjera directa en la economía regional (Honduras, Costa Rica), periodo del 1993 al 2011

Fuente: Datos de los indicadores de Política económica y deuda del Banco Mundial.

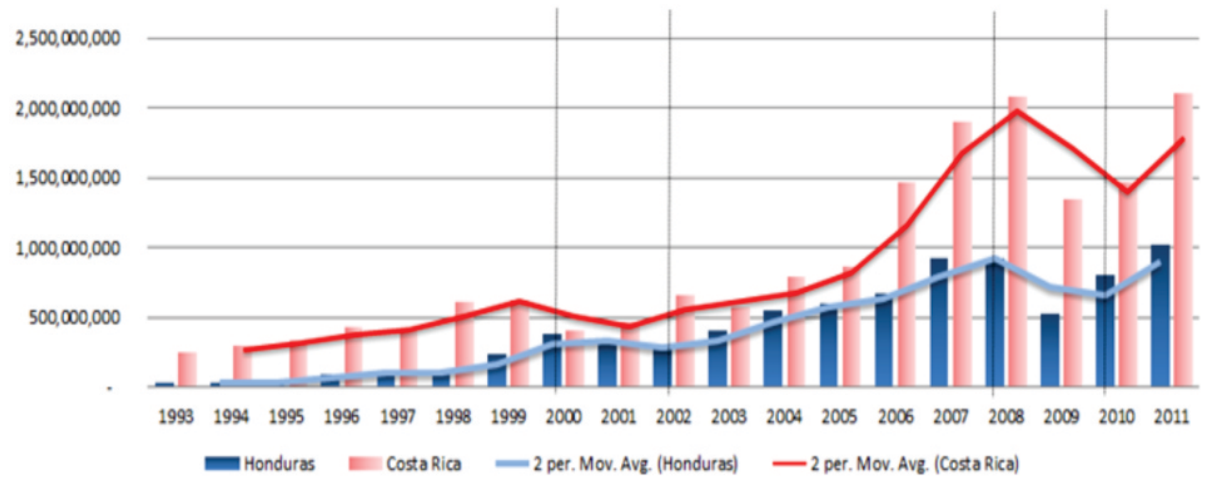

\section{CONSIDERACIONES FINALES}

Del análisis de las estadísticas del Producto Interno Bruto de las economías subdesarrolladas (Honduras y Costa Rica), para con las economías altamente potenciadas y desarrolladas (Alemania, Estados Unidos, Hong Kong) para las series de tiempo de 1993 a 2011, se establece la dependencia directa de Honduras hacia la economía de Estados Unidos de Norte América, diferenciada solo por los problemas políticos, naturales y sociales que se presentaron en Honduras durante los años 1998 y 2009. Por otro lado, Costa Rica tiene un mayor grado de similitud en el comportamiento de sus indicadores macro económicos con la economía de Hong Kong esto a partir de sus oscilaciones y de la revisión de los socios comerciales estratégicos de cada nación. Con la aclaratoria que se debe considerar que las magnitudes de los ingresos y las políticas macro y micro económicas difieren unas de las otras.

Los años donde se muestra el mayor nivel de similitud en el comportamiento del crecimiento del Producto Interno Bruto en los países objeto de estudio es: 19931998, 2000-2001, 2001-2009, 2010-2011. Mostrando mínimos de -5.90, máximos de 8.80, media de 3.24 en el crecimiento mundial. A pesar de ello, los procesos subyacentes de las series económicas no son precisamente lineales, son variables en el tiempo con tendencias diferentes para cada punto analizado. En este sentido, los análisis de las conductas asimétricas del comportamiento económico presentes en la investigación, permiten documentar algunos rasgos ampliamente aceptados entre los que se encuentran el hecho de que las recesiones son más profundas, más 
OSCILACIONES MACROECONÓMICAS DE HONDURAS Y COSTA RICA EN RELACIÓN A LAS ECONOMÍAS DE LOS PAÍSES DESARROLLADOS

Vol. 4

E\&A

IIES

120

volátiles y menos persistentes que las expansiones, visto esto en los gráficos de las oscilaciones de los ciclos económicos de los países en estudio.

En cuanto a la deuda externa acumulada; en Honduras, del año 1994 al 2000 las tasas de crecimiento de la deuda rondaban un promedio de 3\% anual. A pesar de ello, en el año de 2001 cayó a -8\%. Del año 2002 al 2004 la deuda se incrementó a una tasa promedio anual del 7\%, alcanzando su máximo valor (11\%) en 2004. Del 2005 hasta el 2007 los porcentajes adeudados disminuyeron a una tasa promedio del $18 \%$ anual, como resultado de la condonación de deudas y otras estrategias. A partir del año 2008 hasta el 2010 el país se endeudo a una tasa promedio del 8\% anual. En el caso de Costa Rica, a través de la historia muestra niveles de Endeudamiento mayores a los que posee Honduras, con la diferencia que la utilización de los mismos se ha visto en ganancias redituables para el estado y la sociedad. Desde 1994 hasta el año 2010, Costa Rica se ha endeudado a un promedio anual del 5\%, siendo sus picos los años 2005 (14\%), 2007 (20\%), y 2010 (11\%). Del análisis se observa que solo en cuatro años Costa Rica disminuyó su tasa de endeudamiento (1995 (-3\%), $1996(-8 \%), 1997(-1 \%)$ y $2009(-13 \%))$.

En las series de tiempo objeto de análisis, se determinó que las mismas son procíclica debido a que las fases de expansión en cada economía analizada fueron más largas, menos severas y menos volátiles que las fases de contracción, ver crecimiento del PIB, reflejado en las oscilaciones de las tasas de crecimiento de los países en estudio. A su vez, es importante resaltar que los choques externos son importantes en la determinación de las perspectivas de largo plazo para estos países.

Del análisis se resalta el comportamiento de la Inversión Extranjera Directa, ya que ha impulsado, por medio del aumento en las etapas de crisis o recesión, los puntos de giro hacia la recuperación y crecimiento del PIB en cada país objeto de estudio, no obstante, la misma se ve opacada por el incremento del gasto público de las economías tanto desarrolladas como subdesarrolladas. De igual forma, aunque exista acceso a recursos externos para el financiamiento del gasto corriente, si la misma no es bien empleada solo se incrementan los compromisos y se arriesgan las reservas monetarias de las naciones. 
Revista Económica y Administración (E\&A), vol 4(2), pp. 103-122, @ 2013, IIES-UNAH Oscilaciones Macroeconómicas de Honduras y Costa Rica en Relación a las Economías de los Países Desarrollados

\section{BIBLIOGRAFÍA}

Alexander Bobróvnikov, 2002. La Dinámica Ondularia en la Economia Periferica. Amsterdam, Holanda, Univesidad de Holanda, pp. 1-15.

Andrew J. Filardo, 2012. Business-Cycle Phases and Their Transitional Dynamics. Journal of Business \& Economic Statics, pp. 299-308.

Asien, E. R., 2010. Observatorio de la Economía y la Sociedad China. [En línea] Available at: http://www.eumed.net/rev/china/13/era.htm

Banco Mundial, 2012. Grupo del Banco Mundial. [En línea] Available at: http://datos.bancomundial.org/tema/politica-economica-y-deuda BCIE, B. C. d. I. E., 2010. Tendencias \& Perspectivas Económicas de Centroamérica y República Dominicana, Tegucigalpa: s.n.

Burns, Arthur F. and Wesley C. Mitchell, 1946. Measuring Business Cycles. California, United State of America: NBER.

Carcedo, J. M., 2002. Análisis del Ciclo Económico: Descomposición de series temporales, Madrid: Universidad Autónoma de Madrid.

CEPAL Naciones Unidad, 2012. Balance Preliminar de las Economías de América Latina y el Caribe, Mexico: Publicaciones de las Naciones Unidas. CEPAL Naciones Unidas, 2012. Balance Preliminar de las Economias de America Latina y el Caribe / Honduras, Mexico: Publicaciones de las Naciones Unidas .

CEPAL Naciones Unidas, 2012. Informe Macro Economico de America Latina y el Caribe, Chile: Publicaciones de las Naciones Unidas.

Chakravarthi Raghavan, 1995. Tercer Mundo Economico. [En línea] Available at:

http://old.redtercermundo.org.uy/tm_economico/texto_completo.php?id=2189

Clares, A. R., 2003. Análisis del Crecimiento Economico de Costa Rica, San Jose, Costa Rica: Editorial del Banco Interamericano de Desarrollo. Clément Juglar, 1862. Des crises commerciales et de leur retour périodique en France, Angleterre, Etats-Unis. Journal des Economistes, pp. 1-276.

Contreras, F. H., 2009. Observatorio de la Economía y la Sociedad China. [En línea]

Available at: http://www.eumed.net/rev/china/10/cgir.htm

Cordero, J. A., 2009. Honduras: Desempeño Económico Reciente, Washington, United State: Center for Economic and Policy Research.

Fondo Monetario Internacional, 2011. Informe Anual 2011, Buscando un crecimiento equitativo y equilibrado, Washington, Estados Unidos: IMF Publication Services. 
OSCILACIONES MACROECONÓMICAS DE HONDURAS Y COSTA RICA EN RELACIÓN A LAS ECONOMÍAS DE LOS PAÍSES DESARROLLADOS

Vol. 4

E\&A

IIES

Google, 2011. Google. [En línea]

Available at:

http://www.google.hn/publicdata/explore?ds $=\mathrm{d} 5$ bncppjof8f9_\&met_y=sp_pop grow\&idim=country:HND\&dl=es\&hl=es\&q=crecimiento $\% 20 \mathrm{de} \% 201 \mathrm{a} \% 20 \mathrm{pob}$ lacion $\#$ ! ctype $=1 \&$ strail $=$ false $\& b c s=d \& n s e l m=h \&$ met $y=s p \_p o p \_g r o w \&$ scale_y $=$ lin\&ind $\mathrm{y}=$ false $\&$ rdim $=$ region\&idim $=$ country:HND:CRI:USA

Hyman P. Minsky, 1987. Las Razones de Keynes. DF. Mexico : Fondo de Cultura Económica .

Instituto de Estudios Bursatiles, 2011. Estudio de Ciclos Economicos. En: Madrid, España: s.n., pp. 19-71.

Issac Hernandez Contreras, 2009. Observatorio de la Economia y Sociedad

China. [En línea]

Available at: http://www.eumed.net/rev/china/10/cgir.htm Jorba, L. M., 2010. http://www.kascostarica.org. [En línea] Available at: http://www.kascostarica.org/sopla/crecimiento_costarica.pdf Mundo, E., 2010. Desaceleracion de la Economia Alemana, Madrid: s.n. Pablo Bustelo, 1998. Universidad Complutense de Madrid. [En línea] Available at:http://www.ucm.es/info/eid/pb/hongkong98.htm Procesos, 2003. Economia y Desigualda en Costa Rica, San Jose, Costa Rica: Procesos.

Reyes, P. M., 2003. No linealidades y ciclos económicos en América Latina. México : Universidad Autónoma del Estado de México.

Sonia de Lucas Santos, 2006. http://www.ub.edu. [En línea] Available at: http://www.ub.edu/jei/papers/DELGADO-\%20DE\%20LUCASALVAREZ.pdf 